\title{
Modulation of the Work Function in Layer-by-Layer Assembly of Metal Nanoparticles and Poly-L-lysine on Modified Au Surfaces
}

\author{
Michel Carrara, ${ }^{[a, b]}$ Joseph J. Kakkassery, ${ }^{[a]}$ Jean- \\ Pierre Abid, ${ }^{[a]}$ and David J. Fermín ${ }^{*[a, b]}$
}

The electrostatic layer-by-layer assembly of nanoparticles and polyelectrolytes is regarded as one of the most simple and versatile method for the construction of ultrathin organised multilayers. ${ }^{[1-10]}$ The basic protocol is based on the seminal work by Decher on the formation of polyelectrolyte multilayers via al-

[a] Dr. M. Carrara, J. J. Kakkassery, Dr. J.-P. Abid, Prof. Dr. D. J. Fermín Laboratoire d'Electrochimie Physique et Analytique Ecole Polytechnique Fédérale de Lausanne, 1015 Lausanne (Switzerland) E-mail:david.fermin@iac.unibe.ch

[b] Dr. M. Carrara, Prof. Dr. D. J. Fermín

Current address: Departement für Chemie und Biochemie Universität Bern, Freiestrasse 3, 3012 Bern (Switzerland) Fax: (+41) 316-313-994 
ternating deposition of polycations and polyanions. ${ }^{[11,12]}$ The main driving force for the deposition of the colloidal nanoparticles on the polyelectrolyte film is given by the electrostatic interaction between the fixed charges in the outer layer of the film and the charged groups at the particle surface. One of the key advantages of this approach is the possibility of fabricating hybrid materials that incorporate not only metal and semiconductor nanoparticles, but also conducting polymers, ${ }^{[13]}$ mobile redox species ${ }^{[14]}$ and photoactive dyes. ${ }^{[15]}$ Characterisation of polyelectrolyte/nanoparticle assemblies has been performed by a variety of spectroscopic techniques as well as SEM, AFM, conductivity and electrochemical techniques. ${ }^{[4,7,9,10,16-19]}$ However, key aspects such as the structure of the multilayer as a function of the size of the particles remain largely unexplored.

Cant et al. have recently studied Au nanoparticles $(5 \mathrm{~nm}$ diameter) and poly(diallyldimethylammonium) multilayers grown layer-by-layer, by employing Kelvin probe measurements in which oscillating contact potential differences were observed as a function of the number of layers. ${ }^{[20]}$ However, their interpretation based on the Schottky equation commonly used for describing the space charge region in homogeneously doped semiconductor crystals appears to be inappropriate for such disordered systems. Indeed, Liu et al. have shown that similar kind of structures comprising 15 layers of $\mathrm{Au}$ ( $5 \mathrm{~nm}$ diameter) exhibit conductivities of the same order of bulk Au metal. ${ }^{[4]}$ Herein, the structure of metal nanoparticles/poly-L-lysine (PLYS) multilayers grown on modified Au surfaces are studied by Kelvin probe and AFM measurements as a function of the size of the particles. Our previous studies on the generation of two-dimensional assemblies of Au particles of $18 \pm 2 \mathrm{~nm}$ diameter have shown that a maximum number density of particles of $(8.2 \pm 0.1) 10^{10} \mathrm{~cm}^{-2}$ is reached after approximately $4 \mathrm{~h}$ of deposition. ${ }^{[21]}$ The particles are randomly distributed, featuring an average edge-to-edge distance of $25 \mathrm{~nm}$. The formation of this $2 \mathrm{D}$ assembly leads to an increase of the work function $(\Phi)$ with respect to the PLYS terminated surface. The results shown here demonstrate that the modulation of the work function in the layer-by-layer growth of metal nanoparticles and PLYS multilayers is affected not only by the nature but also by the size of the metal particles.

The layer-by-layer assembly of metal nanoparticles and PLYS is initiated by the formation of a self-assembled monolayer (SAM) of 11-mercaptoundecanoic acid (MUA) in ethanol on a freshly evaporated gold film over a period of $12 \mathrm{~h}$. Kelvin probe analysis to be reported in a separate paper shows that the work function steadily increases during the MUA assembling, and reaches an oriented thiol layer after $10 \mathrm{~h}$ of deposition. As shown below (cf. Figure 1), the SAM induces an increase of $\Phi$ by $0.3 \mathrm{eV}$ as a result of changes in the surface dipole. These changes involve dipolar contributions from the gold-thiolate bond as well as the oriented alkyl chain and the carboxy group termination. ${ }^{[22]}$ After consecutive rinsing with ethanol and water, followed by drying in a $\mathrm{N}_{2}$ stream, the modified surface was introduced in an aqueous solution of PLYS at a $\mathrm{pH}$ value of 7 for $30 \mathrm{~min}$. At this $\mathrm{pH}$, the carboxy group of the MUA was deprotonated and the polypeptide was electrostatically adsorbed, which generated a layer approxi- mately 2 to $3 \mathrm{~nm}$ thick. ${ }^{[14]}$ The PLYS-modified surface was subsequently rinsed copiously with water to remove the excess PLYS, followed by drying in a $\mathrm{N}_{2}$ stream, and introduced into the particle colloidal suspension. Based on previous studies on the assembly of citrate-stabilised Au particles, the deposition time was set to $4 h^{[21]}$ The multilayer was grown by alternate deposition of PLYS and the metal particles, with steps of water rinsing and drying after each layer. This protocol generated reproducible two-dimensional layers of $\mathrm{Au}$ nanoparticles in terms of the number density of particles and the spatial distribution. ${ }^{[21]}$

The evolution of $\Phi$ with the number of layers for assemblies of PLYS and Au nanoparticles of diameter ranging from $1.5 \pm$ 0.4 to $19.2 \pm 2.1 \mathrm{~nm}$ is shown in Figure 1. Each Kelvin probe

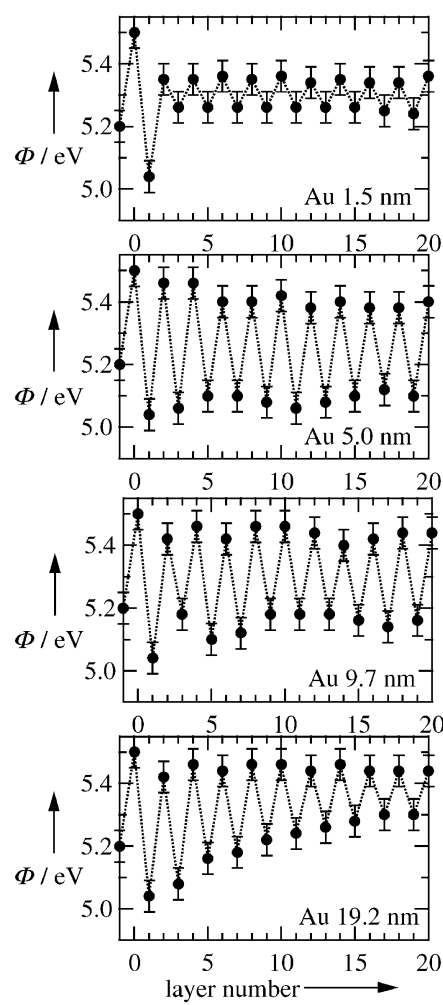

Figure 1. Modulation of the work function in layer-by-layer assembly of gold nanoparticles of various diameters and poly-L-lysine (PLYS). The layer numbers $-1,0$ and 1 correspond to the clean Au substrate, SAM- and PLYS- terminated surfaces, respectively. Even layer numbers are associated with the nanoparticle terminated assembly. The amplitude of modulation is constant as the number of layers increases, except for the Au particles of $19.2 \pm 2.1 \mathrm{~nm}$.

measurement was performed on a freshly prepared sample, which was subsequently disposed of. The layers labelled $-1,0$ and 1 correspond to the freshly evaporated Au film, the MUAand the PLYS-terminated surfaces, respectively. The various sets of curves in Figure 1 show identical values for the steps prior to the nanoparticle deposition, which indicates the reproducibility of the preparation method. In addition, the probe was placed on different areas of the modified surfaces, and the variations of the work function were within the $0.05 \mathrm{eV}$ error margin. A clear modulation of $\Phi$ as a function of the number 
of layers is observed in all the measurements; this behaviour resembles that observed in layer-by-layer growth of some organic composites. ${ }^{[23-25]}$ From classical description of Kelvin probe measurements, the change in the contact potential difference $(U)$ for the surface modification prior to the nanoparticle deposition reflects the change in the surface dipole $(\chi)$ of the system, assuming that the bulk properties of the Au substrate remain unaffected. It follows that, [Equation (1)]

$\Delta U=U_{j}-U_{j-1}=\chi_{j}-\chi_{j-1}$

where the subscript $j$ indicates the layer number. The deposition of a PLYS layer generates a change of the surface dipole, decreasing the energy for electron extraction from the MUAmodified surface. This change in the surface dipole is associated with the distribution of fixed positive charges and counterions in the polycationic film. As discussed further on, the adsorption of the metal nanoparticles involves an equilibration of the Fermi levels, leading to a change of the Volta (outer) potential difference $\psi$. The deposition of a PLYS layer on the nanoparticle-terminated surface induces a new change in the surface dipole, decreasing the work function of the multilayer.

One of the most noticeable trends in the results shown in Figure 1 is the constant amplitude of the modulation of $\Phi$ with the number of layers, except for the array featuring particles of $19.2 \pm 2.1 \mathrm{~nm}$. For the latter assembly, the amplitude decreases as the number of layers increases, which suggests that the regularity in the multilayer structure is affected during the layer-by-layer growth. The noncontact AFM images in Figure 2 reveal a substantial increase in the surface roughness as the layer number increases. This behaviour suggests that the limited thickness of the PLYS layer does not allow a smooth covering of the 2D nanoparticle array, which creates morphological defects that strongly propagate as the number of layers increases. For particles with diameters between $1.5 \pm 0.4$ and $9.7 \pm 1.2 \mathrm{~nm}$, the changes in the surface morphology are not significant within the instrument resolution. The surface roughness, defined as the root-mean-square of the topography, and the average film thickness (measured by a profilometer) for a 20-layer assembly are summarised in Table 1. Although the sur-

\begin{tabular}{|c|c|c|}
\hline Particle diameter [nm] & Surface roughness $[\mathrm{nm}]$ & Thickness [nm] \\
\hline $1.5 \pm 0.4$ & $17 \pm 6$ & $40 \pm 20$ \\
\hline $5.0 \pm 0.7$ & $17 \pm 7$ & $80 \pm 40$ \\
\hline $9.7 \pm 1.2$ & $22 \pm 11$ & $140 \pm 40$ \\
\hline $19.2 \pm 2.1$ & $163 \pm 64$ & $250 \pm 50$ \\
\hline
\end{tabular}

face roughness was estimated from scanned areas of $7 \mu \mathrm{m} \times$ $7 \mu \mathrm{m}$, the values quoted in Table 1 are representative of large portions of the surface. By taking the contribution of each particle layer, the results in Table 1 suggest that the average PLYS layer thickness is between 2.5 and $5 \mathrm{~nm}$. These values are within the range of those reported for PLYS/poly-L-glutamic

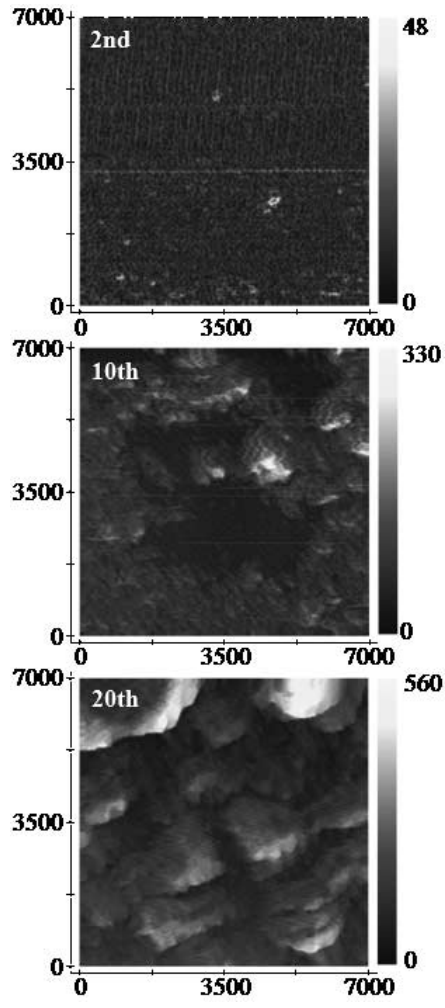

Figure 2. Noncontact AFM pictures of layers number 2, 10 and 20 for multilayers featuring Au nanoparticles of $19.2 \pm 2.1 \mathrm{~nm}$ diameter. All the scales are displayed in nanometers. The substantial increment in the surface roughness coin cides with the decrease in the amplitude of modulation of the work function (Figure 1).

acid multilayers, ${ }^{[14]}$ although the dispersion in the average layer thickness can come as a result of differences in the density of fixed charges in the nanoparticle assembly. The behaviour shown in Figure 1 clearly shows that ordered multilayers can be grown as far as the particle diameter is comparable with the characteristic thickness of the polyelectrolyte layer. Indeed, Early work by Shen and co-workers has shown that electrostatic assembled multilayers featuring $\mathrm{Pbl}_{2}$ particles of $3.8 \mathrm{~nm}$ exhibit well-defined XRD patterns. ${ }^{[26]}$

The amplitude of $\Phi$ modulation is also determined by the polarisability of the deposited layers. In the case of Au particles of $1.5 \pm 0.4 \mathrm{~nm}$, the amplitude of modulation is significantly smaller than for particles of larger diameter. In the case that the oscillations of the work function are determined by the polarisability of the particles, the amplitude is expected to show a cubic dependence on the particle radius. However, the magnitude of the oscillations in Figure 1 is not greatly dependent on the particle size for radii larger than $5 \mathrm{~nm}$. A similar trend (not shown in this work) was observed with multilayers incorporating Ag nanoparticles within the same size range. These results indicate that the amplitude of the surface dipole modulation in multilayers featuring metallic particles larger than $5 \mathrm{~nm}$ is controlled by the polarisability of the polyelectrolyte layer and the degree of layer-by-layer ordering.

Further evidence of the role of the polyelectrolyte on the amplitude of $\Phi$ modulation can be obtained from Figure 3, 


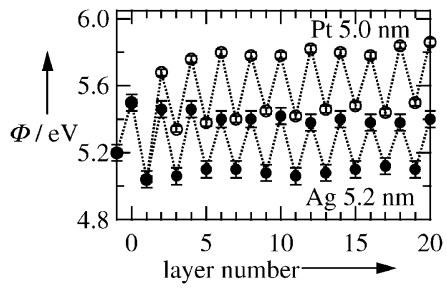

Figure 3. Modulation of the work function for multilayers incorporating $\mathrm{Ag}(\bullet$ and Pt (O) nanoparticles of $5.2 \pm 0.8$ and $5.0 \pm 1.0 \mathrm{~nm}$ diameter, respectively. The modulation amplitude is independent of the nature of the particle, while the work function for the Pt-terminated assemblies is onset by ca. $0.4 \mathrm{eV}$ towards higher values.

which illustrates the oscillating behaviour for multilayers of $\mathrm{Ag}$ and Pt nanoparticles of $5 \mathrm{~nm}$ diameters. It is clearly observed that both multilayered systems exhibit a $0.3 \mathrm{eV}$ modulation of $\Phi$, which also coincides with the value obtained for Au particles of the same size (see Figure 1). An important feature of the results in Figure 3 is the offset of the work function for Pt multilayers towards values higher than that observed for $\mathrm{Au}$ and Ag. The difference in $\Phi$ for the various metal nanoparticles comes as a result of the equilibration of the electrochemical potential of the electrons $\left(\tilde{\mu}_{\mathrm{e}}\right)$, which induces a change in $\psi$ for the particle-terminated surface. The equilibrium condition established between two metals in contact is described by Equation $(2)^{[27]}$

$\tilde{\mu}_{\mathrm{e}}^{\mathrm{mp}}=\tilde{\mu}_{\mathrm{e}}^{\mathrm{ps}}$

therefore, [Equation (3)]

$e\left(\psi^{\mathrm{mp}}-\psi^{\mathrm{ps}}\right)=\Phi^{\mathrm{ps}}-\Phi^{\mathrm{mp}}$

where the superscripts " $\mathrm{mp}$ " and "ps" correspond to the metal nanoparticle and the PLYS-terminated surfaces. It could be further clarified that for assemblies of Au particles, the chemical potential of the electrons in the particles and in the Au substrate can be considered equal and the change in the Volta potential reflects the difference in the surface dipoles associated with the PLYS film and the particle capping group. In this particular case, Equation (3) can be further simplified to Equation (1). The contact potential difference for each of the surface termination is defined as Equations (4) and (5)

$$
\begin{aligned}
& U^{\mathrm{ps}}=\psi^{\mathrm{bc}}-\psi^{\mathrm{ps}} \\
& U^{\mathrm{mp}}=\psi^{\mathrm{bc}}-\psi^{\mathrm{mp}}
\end{aligned}
$$

where $\psi^{\text {bc }}$ is the Volta potential of the back contact of the Kelvin Probe. From Equations (3) to (5), it can be easily derived that the difference in the contact potential for surfaces terminated by two different metal particles corresponds to the difference in their work functions [Equation (6)].

$e\left(U^{\mathrm{mp} 1}-U^{\mathrm{mp} 2}\right)=\Phi^{\mathrm{mp} 1}-\Phi^{\mathrm{mp} 2}$

The results in Figures 1 and 3 show that the work function is $0.4 \mathrm{eV}$ higher for the Pt- than for the Au-terminated surface, which corresponds to the difference in the work functions of the polycrystalline metals. ${ }^{[28]}$ On the other hand, a rather unexpected result arises from the high $\Phi$ values for Ag terminated surface. Considering that $\Phi$ for polycrystalline $\mathrm{Ag}$ is approximately $1 \mathrm{eV}$ lower than $\mathrm{Au}$, the trend illustrated in Figure 3 is expected to be shifted by the same amount towards lower values. In addition, results to be discussed in a separate report show a strong dependence of $\Phi^{\mathrm{Ag}}$ on the size of the Ag nanoparticles, which suggests the presence of an oxide layer on the particle surface. In the case of Au particles, the effect of the particle size on $\Phi^{\mathrm{Au}}$ is rather weak, with a difference of less than $0.1 \mathrm{eV}$ between particles of $1.5 \pm 0.4$ and $19.2 \pm 2.1 \mathrm{~nm}$.

In conclusion, we have provided clear evidences that the work function of metal substrates can be effectively controlled by electrostatic assembly of metal nanoparticles. In layer-bylayer assemblies featuring poly-L-lysine, the modulation of $\Phi$ is affected by the particle size for particle diameters below $5 \mathrm{~nm}$. For larger particles, the amplitude of modulation is determined by the polarisability of the ultrathin polyelectrolyte film and the layer-by-layer ordering. The value of the $\Phi$ for the nanoparticle-terminated surface is dependent on the work function of the particle. This result indicates that the Fermi level in the particle is in equilibrium with the metal substrate. Consequently, it can be envisaged that the work function of the assembly can be tuned over a wide range of values by employing particles of different nature, or by changing the capping group at the particle surface. These results open up the possibility of designing junctions with rectifying properties, which can be key elements for the development of hybrid optoelectronic ${ }^{[29]}$ and photovoltaic devices. ${ }^{[3,30]}$

\section{Experimental Section}

Synthesis of Nanoparticles: Gold nanoparticles with diameters larger than $5 \mathrm{~nm}$ were synthesised by reduction of the metal cation precursor by employing trisodium citrate. The Au precursor employed in all the syntheses was $\mathrm{HAuCl}_{4} \cdot 3 \mathrm{H}_{2} \mathrm{O}$. The solution of the metal cation was refluxed under vigorous stirring prior to the addition of citrate. To control the size of the nanoparticles, the concentration ratio of the precursor to citrate was systematically varied from 1:5 $(5.0 \pm 0.7 \mathrm{~nm}), \quad 1: 4.2(9.7 \pm 1.2 \mathrm{~nm})$ and 1:0.62 $(19.2 \pm 2.1 \mathrm{~nm}){ }^{\left[{ }^{[28]}\right.}$ After the introduction of citrate, the reduction process was extended over $45 \mathrm{~min}$ at boiling point. $\mathrm{Ag}$ and $\mathrm{Pt}$ nanoparticles were obtained by reduction of $\mathrm{AgNO}_{3}$ and $\mathrm{H}_{2} \mathrm{PtCl}_{6}$ using $\mathrm{NaBH}_{4}$ in 1:1 ratio of the metal cation and citrate. Au nanoparticles of $1.5 \pm 0.4 \mathrm{~nm}$ diameter were prepared by reduction of the metal precursor in the presence of $\mathrm{NaBH}_{4}$ and mercaptosuccinic acid as an electrostatic stabiliser. Details of the synthetic method have been published elsewhere. ${ }^{[27,28]}$

Gold substrate preparation: The nanoparticle/poly-L-lysine (MW 39000 (vis) Sigma) multilayers were grown on evaporated Au surface modified by a self-assembled monolayer of 11-mercaptoundecanoic acid (Aldrich). The gold films were evaporated on glass slides at a pressure of less than $5 \times 10^{-6} \mathrm{mbar}$. The slides were cleaned by sequential sonication in ethanol, acetone and purified water, followed by drying under a stream of $\mathrm{N}_{2}$. The film growth was initiated by the evaporation of a $10 \AA$ thick chromium layer at $1 \AA s^{-1}$ to enhance the adhesion of the Au layer. Gold was subsequently evaporated at $1 \AA s^{-1}$ up to $50 \AA$, which increased the dep- 
osition rate to $20 \AA \mathrm{s}^{-1}$ for depositing a layer of $1000 \AA$ thick of amorphous gold with a work function of $5.20 \pm 0.05 \mathrm{eV}$.

Instrumentation: Measurements of the work function were performed with a Kelvin probe (Delta Phi) in a glove box under $\mathrm{N}_{2}$ atmosphere and at room temperature in the dark. A gold plate was employed as a reference electrode, which was kept at less than $1 \mathrm{~mm}$ distance from the sample. The AFM measurements were performed in noncontact mode employing a Burleigh Personal SPM. The thickness of the multilayers was measured by Alpha-Step 500 Surface Profiler. The nanoparticles sizes were obtained from Transmission Electron Microscopy employing a Philips CM20.

\section{Acknowledgements}

We are grateful to Prof. Libero Zuppiroli and Dr. Frank Nüesch from the Laboratoire d'Optoélectronique des Matéraiux Moléculaires of the EPFL for the access to the Kelvin probe facilities. We acknowledge the Centre Interdépartemental de Microscopie Electronique of the EPFL for the access to the TEM facilities. The discussions and support by Prof. Hubert Girault as well as the technical support by Valérie Devaud are also gratefully acknowledged. This work was funded by the Swiss National Foundation (projects 2000-067050.01 and PP002-60708/1).

Keywords: metal-metal interactions • nanostructures polyelectrolytes $\cdot$ self-assembly $\cdot$ work functions

[1] X. Zhang, J. C. Shen, Adv. Mater. 1999, 11, 1139.

[2] A. N. Shipway, E. Katz, I. Willner, ChemPhysChem 2000, 1, 18

[3] N. A. Kotov, I. Dekany, J. H. Fendler, J. Phys. Chem. 1995, 99, 13065.

[4] Y. J. Liu, Y. X. Wang, R. O. Claus, Chem. Phys. Lett. 1998, 298, 315.

[5] J. F. Hicks, Y. Seok-Shon, R. W. Murray, Langmuir 2002, 18, 2288.

[6] N. Ferreyra, L. Coche-Guerente, J. Fatisson, M. L. Teijelo, P. Labbe, Chem. Commun. 2003, 2056

[7] T. Sagara, N. Kato, N. Kakashima, J. Phys. Chem. B 2002, 106, 1205.

[8] K. M. Chen, X. P. Jiang, L. C. Kimerling, P. T. Hammond, Langmuir 2000, 16,7825 .

[9] K. Uosaki, T. Kondo, M. Okamura, W. Song, Faraday Discuss. 2002, 121, 373.

[10] W. Song, M. Okamura, T. Kondo, K. Uosaki, J. Electroanal. Chem. 2003, 554-555, 385

[11] G. Decher, Science 1997, 277, 1232

[12] G. Decher, Photonics Optoelectron. 1997, 672, 445.

[13] T. Cassagneau, T. E. Mallouk, J. H. Fendler, J. Am. Chem. Soc. 1998, 120, 7848

[14] Y. Cheng, R. M. Corn, J. Phys. Chem. B 1999, 103, 8726.

[15] J. J. Kakkassery, D. J. Fermin, H. H. Girault, Chem. Commun. 2002, 1240.

[16] G. Bogdanovic, T. Sennerfors, B. Zhmud, F. Tiberg, J. Colloid Interface Sci. 2002, 255, 44.

[17] H. S. Kim, B. H. Sohn, W. Lee, J. K. Lee, S. J. Choi, S. J. Kwon, Thin Solid Films 2002, 419, 173.

[18] T. Sennerfors, G. Bogdanovic, F. Tiberg, Langmuir 2002, 18, 6410

[19] T. Sagara, N. Kato, A. Toyota, N. Nakashima, Langmuir 2002, 18, 6995.

[20] N. E. Cant, H.-L. Zhang, K. Critchley, T. A. Mykhalyk, G. R. Davies, S. D. Evans, J. Phys. Chem. B 2003, 107, 13557.

[21] J. J. Kakkassery, J.-P. Abid, M. Carrara, D. J. Fermín, Faraday Discuss. 2004, 125, 157.

[22] S. D. Evans, A. Ulman, Chem. Phys. Lett. 1990, 170, 462.

[23] L. S. Li, A. D. Q. Li, J. Phys. Chem. B 2001, 105, 10022

[24] L. S. Li, R. Wang, M. Fitzsimmons, D. Q. Li, J. Phys. Chem. B 2000, 104, 11195.

[25] L. S. Li, Q. X. Jia, A. D. Q. Li, Chem. Mater. 2002, 14, 1159.

[26] M.-Y. Gao, M. Gao, X. Zhang, Y. Yang, B. Yang, J. C. Shen, Chem Commun. 1994, 2777
[27] M. Fujihira, Annu. Rev. Mater. Sci. 1999, 29, 353

[28] S. Trasatti, in Comprehensive Treatise of Electrochemistry, Vol. 1 (Eds.: J. O. M. Bockris, B. E. Conway, E. Yeager), Plenum Press, N. Y., 1980, pp. 45.

[29] S. Coe, W. K. Woo, M. Bawendi, V. Bulovic, Nature 2002, 420, 800.

[30] D. E. Fogg, L. H. Radzilowski, B. O. Dabbousi, R. R. Schrock, E. L. Thomas, M. G. Bawendi, Macromolecules 1997, 30, 8433

Received: December 19, 2003 [200301212] 